\title{
Multicolor photometry of the Vela pulsar ${ }^{\star \star \star}$
}

\author{
R. P. Mignani ${ }^{1}$ and P. A. Caraveo ${ }^{2}$ \\ 1 ESO, Karl Schwarzchild Str. 2, 85740 Garching bei München, Germany \\ 2 Istituto di Fisica Cosmica del CNR "G. Occhialini”, Via Bassini 15, 20133 Milan, Italy
}

Received 2 March 2001 / Accepted 29 June 2001

\begin{abstract}
Multicolor photometry of the Vela pulsar (PSR B0833-45), updated by recent HST/WFPC2 observations obtained in the $555 \mathrm{~W}, 675 \mathrm{~W}$ and $814 \mathrm{~W}$ filters, is presented. The available data provide the best characterization so far of the pulsar spectral shape, which is dominated by a flat power law continuum with spectral index $\alpha=-0.2 \pm 0.2$, consistent with the extrapolation in the optical domain of the power law component of the X-ray spectrum detected by CHANDRA. In addition, a marginally significant dip $(\approx 3 \sigma)$ seems to be present at about 6500 Å. Spectroscopic observations with the VLT, now in progress, will undoubtly provide a much better assessment of the reality and characteristics of this feature.
\end{abstract}

Key words. pulsars: individual: PSR B0833-45

\section{Introduction}

The Vela pulsar (PSR B0833-45) has been the first isolated neutron star to be identified in the optical after the Crab pulsar. Its optical counterpart $(V=23.6)$, originally discovered by Lasker (1976), was found to pulsate at the radio period $(89 \mathrm{~ms}$ ) by Wallace (1977) with a sharp double-peaked light curve. With an optical luminosity of about $10^{28} \mathrm{erg} \mathrm{s}^{-1}$, the Vela pulsar is the third brightest isolated neutron star after the Crab pulsar and PSR B0540-69, albeit with a much lower optical efficiency as indicated in the work of Goldoni et al. (1997).

Too old to be counted among the young pulsars $(\approx 1000-5000$ yrs) and too young to belong to the middleaged class $(\geq 100000 \mathrm{yrs})$, Vela ( $\approx 10000 \mathrm{yrs}$ ) appears as a transition object between the two groups of neutron stars, which are characterized by very different emission properties both in the optical and in the X-ray regime. While in the young pulsars pure synchrotron radiation (e.g. Pacini 1971) yields a relatively high optical emission efficiency and power law spectra (see e.g. Hill et al. 1997; Sollerman et al. 2000), different emission mechanisms appear at work in older objects. Indeed, PSR B0656+14 (Pavlov et al. 1997; Koptsevich et al. 2001) and Geminga

Send offprint requests to: R. P. Mignani, e-mail: rmignani@eso.org

* Based on observations with the NASA/ESA Hubble Space Telescope, obtained at the Space Telescope Science Institute, which is operated by AURA, Inc. under contract No. NAS $5-26555$.

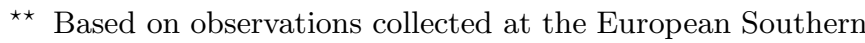
Observatory, La Silla, Chile.
(Martin et al. 1998; Mignani et al. 1998) show more complex spectral shapes, coupling magnetospheric and thermal radiation with a definitely lower emission efficiency. It is generally agreed that, as the pulsars age and slow down, the magnetospheric component of the optical emission becomes progressively weaker, while the thermal one becomes more significant and finally dominates (van Kerkwijk \& Kulkarni 2001). A similar evolution has been observed in the soft X-ray regime, where young pulsars exhibit pure magnetospheric, strongly pulsed emission (Becker \& Trümper 1997) while the radiation from middle-aged neutron stars is well described by a black body emission with shallow modulations. Vela is a unique example of a mostly thermal soft X-ray emitter (e.g. Pavlov et al. 2001), with just a few percent modulation, producing also totally pulsed optical emission (see, e.g., Gouiffes et al. 1998), albeit with a low efficiency (Goldoni et al. 1997). Thus, characterizing its optical spectrum would be important to trace the evolution in the emission physics between the two groups of young and middle-aged neutron stars.

So far, the most complete optical spectral study of the Vela pulsar has been carried out by Nasuti et al. (1997) through ground-based $U B V R$ band photometry. Its multicolor flux distribution was found to lie above any realistic blackbody curve associated with the neutron star's surface emission (Ögelman et al. 1993), which clearly confirmed its magnetospheric origin. However, Nasuti et al. showed that the Vela pulsar spectrum was substantially different from that of the Crab pulsar and of PSR B0540-69. The main difference was due to a decrease in the flux in the 
$R$ band, which was taken by Nasuti et al. as an indication for either a spectral turnover at a wavelength $\geq 6000 \AA$ or an absorption feature.

Since the lack of spectral information at longer wavelenghts was the main limit for a complete characterization of the Vela pulsar spectrum, we have performed new accurate photometry observations close to the $R$ and $I$ bands with the WFPC2 on HST. A description of the observations and of the data reduction is given in Sect. 2 while the results are discussed in Sect. 3 .

\section{Observations and data reduction}

The Vela pulsar was observed with the WFPC2 aboard the HST on March 15th and 19th 2000. The observations were performed through the filters $675 \mathrm{~W}(\lambda=6717 \AA$, $\Delta \lambda=1536 \AA)$ and $814 W(\lambda=7995 \AA, \Delta \lambda=1292 \AA)$. While the observation in the $675 W$ filter was aimed at confirming the flux value of Nasuti et al. in the $R$ band, the observation through the $814 \mathrm{~W}$ one was taken to disentangle the presence of an absorption dip from a real spectral turnover. In addition, WFPC2 images of the Vela pulsar were repeatedly obtained with the $555 \mathrm{~W}$ filter $(\lambda=5442 \AA, \Delta \lambda=1044 \AA)$ as a part of a set of observations aimed at the measurement of the neutron star's parallax (Caraveo et al. 2001). In all cases, the exposure time was set to $2600 \mathrm{~s}$, corresponding to a single orbit of the spacecraft. The pulsar was always located at the center of the Planetary Camera (PC) chip of the WFPC2, with a corresponding pixel size of 45.5 mas and a field of view of $35 \times 35$ arcsec. All the observations were split into shorter exposures to allow for a better cosmic ray filtering. After the standard pipeline processing to remove instrumental effects, single exposures were combined using a median filter algorithm. Flux calibration was computed following the standard WFPC2 recipe (e.g. Holtzmann et al. 1995). The pulsar was clearly detected in all passbands and its magnitude was computed by standard aperture photometry using the same optimized areas to estimate the counts both from the source and from the sky background. The magnitudes in the three passbands $814 \mathrm{~W}$, $675 \mathrm{~W}$ and $555 \mathrm{~W}$ were thus computed to be $24.40( \pm 0.1)$, $24.30( \pm 0.1)$ and $23.64( \pm 0.05)$, respectively, with the photometric error in the $555 \mathrm{~W}$ filter being averaged over the five epochs.

\section{Results}

The complete multicolor photometry of the Vela pulsar, including both the newly acquired HST values and the previous ground based measurements of Nasuti et al. (1997), is summarized in Table 1. Magnitudes have been corrected for the interstellar extinction using as a reference $A_{V}=0.4$, as reported in Manchester et al. (1978). The corresponding flux values are plotted in Fig. 1, where the HST data are seen to improve significantly on the groundbased colours of Nasuti et al. In particular, the spectrum shown in Fig. 1, with the first flux measurement in the

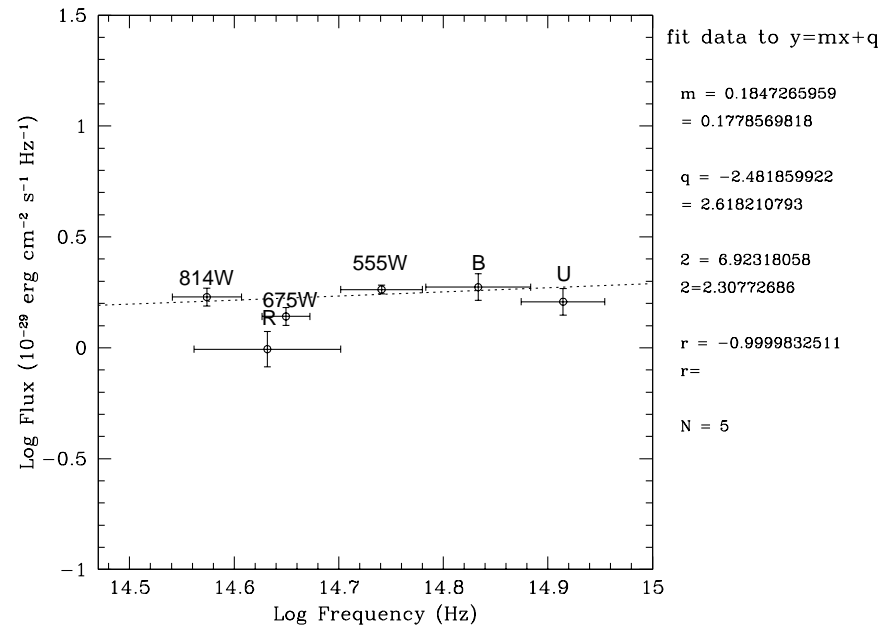

Fig. 1. Multicolor fluxes of PSR 0833-45 computed from the magnitudes listed in Table 1. The $U B V R$ fluxes have been obtained by the NTT (Nasuti et al. 1997), while the ones labelled with the three digits $(814 \mathrm{~W}, 675 \mathrm{~W}$ and $555 \mathrm{~W})$ have been obtained with the HST/WFPC2. The $V$ point virtually coincides with the $555 \mathrm{~W}$ one and is not plotted for clarity. The dashed line represents the power law $(\alpha=-0.2 \pm 0.2)$ best fitting the available data set with the exception of the $R$ point, which appears to deviate from the trend and suggests the possible presence of a dip around $6500 \AA$.

$I$-band, provides no indication for the spectral turnover suggested by Nasuti et al. on the basis of their limited data set. The spectral distribution is compatible with a power law spectrum $\left(F_{\nu} \propto \nu^{-\alpha}\right)$, as expected in the case of pure magnetospheric radiation (Pacini 1971). A fit to the points shown in Fig. 1, with the exception of the clearly deviant $R$ point, gives a spectral index $\alpha=-0.2 \pm 0.2$, which is comparable with that of the Crab pulsar $(\alpha=0.1 \pm 0.01)$ obtained from spectroscopic observations by Nasuti et al. (1996) and more recently confirmed by Sollerman et al. (2000). In particular, is interesting to note that the slope of the Vela spectral distribution is consistent with the extrapolation of the power law magnetospheric component measured by CHANDRA (Pavlov et al. 2001) for $E \geq 1.8 \mathrm{keV}$. This points towards a common origin for both the optical and the X-ray emissions.

Although the optical spectrum of Vela is dominated by a flat continuum, a deviation from the trend can be seen around $6500 \AA$, where the $675 \mathrm{~W}$ point seems to follow the trend for a flux decrease suggested by the ground based, less accurate, $R$ point. On the other hand, the $814 \mathrm{~W}$ measurement is higher than both the $R$ and $675 \mathrm{~W}$ ones. Applying different reddening corrections to our data using, e.g. the $N_{\mathrm{H}}$ obtained from the spectral fits to the ROSAT (Ögelman 1993; Page et al. 1996) or CHANDRA (Pavlov et al. 2001) data does not substantially alter our result. Thus, the newly acquired colours suggest the presence of a dip in the spectrum at $\approx 6500 \AA$. Within the uncertainty of our photometry and of the flux calibration, we can estimate its significance at about $\approx 3 \sigma$. However, given the marginal statistical weight of this detection, 
Table 1. Summary of the available multicolor photometry of the Vela pulsar. The telescopes and detectors used, together with the observing epochs, are listed in the first three columns, respectively. The filter names are reported in the fourth column while their pivot wavelength and width are in the fifth. The observed magnitudes, computed wrt to the Johnson and HST photometric systems, and attached errors are listed in column six. Column seven gives the corresponding fluxes and errors at the pivot wavelengths after dereddening for an $A_{V}=0.4$ (Manchester et al. 1978). The $555 W$ magnitude is the average of independent measurements obtained at five different epochs.

\begin{tabular}{c|c|c|c|c|c|c}
\hline Tel & Instrument & Date & Filter & Wavelength/Width & mag & $\begin{array}{c}\text { Flux } \\
\left(10^{-29} \mathrm{erg} \mathrm{cm}^{-2} \mathrm{~s}^{-1} \mathrm{~Hz}^{-1}\right)\end{array}$ \\
\hline HST & WFPC2 & 19.03 .2000 & $814 W$ & $7995 \AA(1292 \AA)$ & $24.40(0.1)$ & $1.69(0.16)$ \\
NTT & EMMI & 30.01 .1995 & $R$ & $6410 \AA(1540 \AA)$ & $23.93(0.2)$ & $0.98(0.20)$ \\
HST & WFPC2 & 15.03 .2000 & $675 W$ & $6717 \AA(1536 \AA)$ & $24.30(0.1)$ & $1.38(0.13)$ \\
NTT & EMMI & 30.01 .1995 & $V$ & $5426 \AA(1044 \AA)$ & $23.65(0.1)$ & $1.84(0.16)$ \\
HST & WFPC2 & $(*)$ & $555 W$ & $5442 \AA(1044 \AA)$ & $23.64(0.05)$ & $1.82(0.08)$ \\
NTT & EMMI & 30.01 .1995 & $B$ & $4223 \AA(941 \AA)$ & $23.89(0.15)$ & $1.88(0.26)$ \\
NTT & EMMI & 30.01 .1995 & $U$ & $3542 \AA(542 \AA)$ & $23.38(0.15)$ & $1.61(0.22)$ \\
\hline
\end{tabular}

(*) 30.06.1997, 2.01.1998, 30.06.1999, 15.01.2000, 5.07.2000.

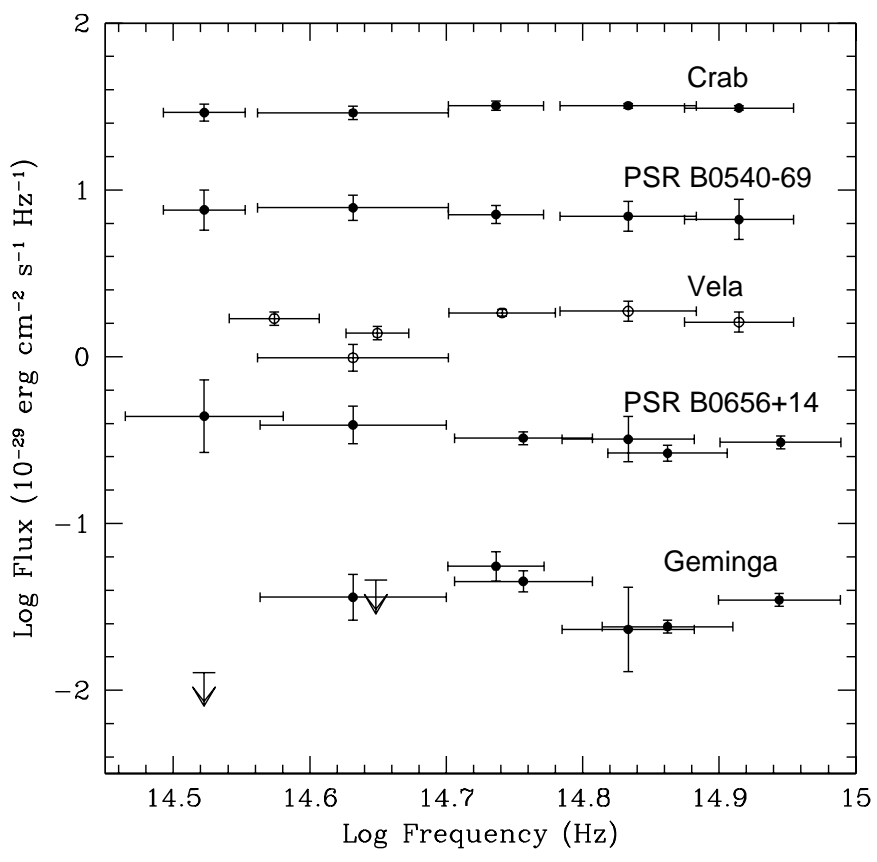

Fig. 2. The $I$ to $U$ colors of the Vela pulsar (open circles) are compared to those of the other optically emitting isolated neutron stars (filled circles). From top to bottom: the Crab pulsar and PSR B0540-69 (Nasuti et al. 1997), PSR 0656+14 and Geminga (Koptsevich et al. 2001; Mignani et al. 1998). To allow for a better representation on the same frame, the fluxes of the Crab pulsar and Geminga have been re-scaled.

admittedly affected by the lower accuracy of the groundbased $R$ point, any interpretation of the physical origin of the dip would be premature.

The spectral distribution of the Vela pulsar can be compared with those of the other isolated neutron stars with multicolor photometry. This is done in Fig. 2, where the objects' fluxes have been scaled to fit into the same frame. Although limited to five objects (the Crab pulsar, PSR B0540-69, the Vela pulsar, PSR B0656+14 and Geminga), this sample covers more than two decades in pulsar age and ten magnitudes in flux going all the way from the bright, 1000 yrs old, Crab to the $25.5 \mathrm{mag}$, 500000 yrs old, Geminga (see, e.g., Caraveo 2000 for a recent review). From the youngest to the oldest, the flux distributions increase in complexity going from monotonic, power-law spectra to composite ones, where the thermal emission from the hot neutron star surface adds to the magnetospheric one (Mignani et al. 1998; Pavlov et al. 1997). Such an evolutionary interpretation is proposed also by Koptsevich et al. (2001) who, on the basis of a similar comparative multicolor study aimed at putting in context their new results on PSR B0656+14, remark that the non monotonic behaviour seen both for Geminga and PSR 0656+14, "might indicate the presence of unresolved spectral features". While waiting for spectroscopic observations of more isolated neutron stars, we can safely state that the spectral distributions measured for middleaged isolated neutron stars look different from that of the very young ones, underlining the key position of the Vela pulsar.

Both age-wise and flux-wise, Vela appears to occupy an "in between" position. Its flat, power-law spectrum, coupled with its $100 \%$ pulsation, makes it similar to the younger objects, while its low efficiency brings it nearer to the older ones.

\section{Conclusions}

We have reported new colours of the Vela pulsar in the red part of the spectrum, obtained with the HST/WFPC2. These observations, which also provide the first detection of the pulsar in the $I$ band, have been used to improve and complement the previous ground-based results of Nasuti et al. (1997). The pulsar spectral distribution, now determined with unprecedented detail, is clearly dominated by a flat power law continuum $(\alpha=-0.2 \pm 0.2)$ with no spectral turnover. In addition, a marginal indication for a dip is found at $\approx 6500 \AA$. The first spectroscopic observations of the Vela pulsar, now in progress with the VLT, are needed to confirm the reality of such a dip and to address its origin. 
Acknowledgements. We wish to thank the anonymous referee for his/her comments, which helped to improve the quality of the paper.

\section{References}

Caraveo, P. A., DeLuca, A., Mignani, R. P., \& Bignami, G. F. 2001, ApJ, in press [astro-ph/0107282]

Caraveo, P. A. 2000, Proc. Pulsar Astronomy- 2000 and Beyond, ed. M. Kramer, N. Wex, \& R. Wielebinski, ASP Conf. Ser., 202, 289

Goldoni, P., Musso, C., Caraveo, P. A., \& Bignami, G. F. 1995, A\&A, 298, 535

Gouiffes, C. 1998, Proc. Neutron Stars and Pulsars: 30 years after the discovery, ed. N. Shibazaki, N. Kawai, S. Shibata, \& T. Kifune, 363 (Universal Academic Press)

Holtzmann, J. A., Burrows, C. J., Casertano, S., et al. 1995, PASP, 107, 1065

Hill, R. J., Dolan, J. F., Bless, R. C., et al. 1997, ApJ, 486, L99

Koptsevich, A. B., Pavlov, G. G., Zharikov, S. V., et al. 2001, A\&A, 370, 1004

Lasker, B. M. 1976, ApJ, 203, 193
Manchester, R. N., et al. 1978, MNRAS, 184, 159

Martin, C., Halpern, J. P., \& Schiminovich, D. 1998, ApJ, 494, L211

Mignani, R., Caraveo, P. A., \& Bignami, G. F. 1998, A\&A, 332, L37

Nasuti, F. P., Mignani, R., Caraveo, P. A., \& Bignami, G. F. 1996, A\&A, 314, 849

Nasuti, F. P., Mignani, R., Caraveo, P. A., \& Bignami, G. F. 1997, A\&A, 323, 839

Ögelman, H., Finley, J. P., \& Zimmermann, H. U. 1993, Nature, 361, 136

Pacini, F. 1971, ApJ, 163, L17

Page, D., Shibanov, Y. A., \& Zavlin, V. E. 1996, Proc. Roentgenstrahlung from the Universe, ed. H. U. Zimmermann, J. Trümper, \& H. Yorke, 173

Pavlov, G. G., Welty, A. D., \& Cordova, F. A. 1997, ApJ, 489, L75

Pavlov, G. G., Zavlin, V. E., Sanwal, D., Burwitz, V., \& Garmire, G. P. 2001, ApJ, 552, L129

Sollerman, J., Lundqvist, P., Lindler, D., et al. 2000, ApJ, 537, 861

van Kerkwijk, M. H., \& Kulkarni, S. R. 2001, A\&A, submitted [astro-ph/0106265]

Wallace, P. T., et al. 1977, Nature, 266, 692 